\title{
Astrophysical Measurement of the Equation of State of Neutron Star Matter
}

\author{
Feryal Özel, ${ }^{1}$ Gordon Baym, ${ }^{2}$ and Tolga Güver ${ }^{1}$ \\ ${ }^{1}$ University of Arizona, Department of Astronomy and Steward Observatory, 933 N. Cherry Ave., Tucson, AZ 85721 \\ ${ }^{2}$ Department of Physics, University of Illinois, 1110 W. Green St., Urbana, IL 61801
}

\begin{abstract}
We present the first astrophysical measurement of the pressure of cold matter above nuclear saturation density, based on recently determined masses and radii of three neutron stars. The pressure at higher densities are below the predictions of equations of state that account only for nucleonic degrees of freedom, and thus present a challenge to the microscopic theory of neutron star matter.
\end{abstract}

PACS numbers: 97.60.Jd, 26.60.Kp, 21.65.-f

Neutron stars probe the dense QCD phase diagram at lower temperatures and higher baryon densities, in contrast to the higher temperature-lower density regime in the early universe and in ultrarelativistic heavy-ion collisions [1, 2]. The baryon density, $\rho$, in stellar interiors may reach an order of magnitude beyond nuclear saturation density, $\rho_{\mathrm{ns}} \simeq 2.7 \times 10^{14} \mathrm{~g} \mathrm{~cm}^{-3} \simeq 0.16 \mathrm{fm}^{-3}$, and cross into a regime where quark degrees of freedom are excited or matter undergoes a meson condensation phase transition. The pressure of matter at these high densities can, as we show here, be extracted from current neutron star mass-radius determinations [3], and crucially constrains calculations of high density neutron star matter.

The equation of state (EoS) of supranuclear matter determines the dividing line between neutron stars and black holes, and directly impacts the mechanism as well as outcomes of supernova explosions and the numbers of neutron stars and black holes in the Galaxy [4, 5]. In certain models, it affects the mechanism and duration of gamma-ray bursts [6]. Accurate evolutions of inspiraling neutron-star binaries and the collapse to black holes, needed to calculate gravitational wave signals, depend sensitively on the assumed EoS 7].

Microscopic calculations of the EoS of neutron-star matter have been based on a variety of inputs. The approach most firmly founded on experiment in the region of $\rho_{\mathrm{ns}}$ is to determine two-body potentials from nucleonnucleon scattering data below $350 \mathrm{MeV}$ and properties of light nuclei, supplemented by a three-body potential [8, 9]. Such calculations, accurate in the neighborhood of $\rho_{\text {ns }}$, have fundamental limitations. Beyond a few times $\rho_{\mathrm{ns}}$ the forces between particles can no longer be described via static few-body potentials; since the characteristic range of the nuclear forces is $\sim 1 / 2 m_{\pi}$, where $m_{\pi}$ is the mass of the pion, the parameter measuring the relative importance of three and higher body forces is $\sim \rho /\left(2 m_{\pi}\right)^{3} \sim 0.35 \rho / \rho_{\text {ns }}$. Thus, at $\rho \gg \rho_{\text {ns }}$ a well defined expansion in terms of two-, three-, or more, body forces no longer exists. EoS based on nucleons alone do not take into account the rich variety of hadronic degrees of freedom that enter with increasing density. In addition, pion condensates [8, 10, 11] or kaon condensates [12, 13] can enter at higher densities. Field-theoretic models based on nucleons interacting via meson exchange include, e.g., Ref [14]; see Ref. [15] for a general summary of EoS. How- ever, one cannot assume that matter at higher densities can even be described in terms of well-defined "asymptotic" laboratory particles. More realistically, one expects in dense matter a gradual onset of quark degrees of freedom, not accounted for by nucleons interacting via static potentials. Indeed once nucleons overlap considerably, the matter should percolate, opening the possibility of their quark constituents propagating throughout the system. Such additional degrees of freedom should lead to softening of the EoS, consistent with our findings here, and thus to a lower maximum neutron star mass. Owing to difficulties of determining the neutron star matter EoS from first principles, neutron star observations become an important input in determining the EoS at high density, and constraining microscopic calculations.

A number of astrophysical observations have focused on measuring neutron star radii, $R$, and masses, $M$, in an attempt to constrain the uncertainties in the EoS (summarized in Ref. [15]). The measurement of postNewtonian parameters of double neutron stars provide precise determinations of their masses [16]. Glitches observed in radio pulsars lead to constraints on the moment of inertia, and therefore, on neutron star masses and radii [17]. Observations of the thermal emission from accreting neutron stars in quiescence and from millisecond X-ray pulsars result in broad, correlated constraints on neutron star masses and radii [18].

Recent advances in both astrophysical techniques and neutron-star atmosphere modeling allow us for the first time to measure the pressure of neutron star matter at supranuclear densities directly from observations. References [15] suggested that the radii of neutron stars are a good indicator of the pressure at roughly twice the nuclear saturation density. This argument was further extended in 19, 20], which demonstrated that knowing the properties of ultradense matter at three fiducial densities allows one to reproduce macroscopic neutron star properties, including the mass-radius relation and stellar moment of inertia. Conversely, three distinct measurements of neutron star masses and radii, as we use here, is sufficient to infer a piecewise polytropic EoS of matter at supranuclear densities [19].

Observations of multiple spectroscopic phenomena during thermonuclear bursts from X-ray binaries have enabled the tightest measurements neutron star radii 
and masses to date [3]. The long-term monitoring of burst sources with the Rossi X-ray Timing Explorer, with its excellent photon statistics, has resulted in a large (> 1000) database of bursts [21], from which systematic uncertainties can be determined and controlled, and ideal sources that act as standard candles can be identified. High resolution X-ray spectroscopy with the Chandra Xray Observatory and XMM-Newton has led to a detailed measurement of the soft X-ray spectra of bursters and reduced the uncertainties introduced by interstellar extinction [22]. Finally, pointed optical/infrared observations with the Hubble Space Telescope and large ground-based facilities (e.g., the Magellan telescope) have substantially improved distance measurements to these sources (see Ref. 3] and references therein).

The wealth of such high quality data allows us to employ a novel approach, combining different spectroscopic measurements to break the degeneracies between neutron star masses and radii inherent to each observable 23, 24]. The first observable is the apparent surface area during the cooling phase of the bursts,

$$
A=\frac{R^{2}}{D^{2} f_{\mathrm{c}}^{4}}\left(1-\frac{2 G M}{R c^{2}}\right)^{-1}
$$

where $D$ is the distance to the source, and $f_{\mathrm{c}}$ is a calculated ratio between the spectral (color) and effective temperature, $T$, of the emerging radiation that accounts for the non-Planckian spectrum of the burst. Because the emitted luminosity is $\propto T^{4}$, the apparent surface area in Eq. (11) shows the same $T$ dependence, which we absorb into the definition of $f_{\mathrm{c}}$. The apparent surface area remains constant in time and is highly reproducible in multiple events from the same source, indicating that the entire neutron star surface, rather than a variable area on the surface, participates in the burst emission.

The second phenomenon occurs in a subset of bursts, when the flux becomes so high that it exceeds the local Eddington limit and lifts the photosphere of the neutron star. The flux achieved during these events is also highly reproducible for a large number of sources including the three discussed below, and is related to the neutron star mass and radius through

$$
F_{\mathrm{Edd}}=\frac{G M c}{k_{\mathrm{es}} D^{2}}\left(1-\frac{2 G M}{R c^{2}}\right)^{1 / 2} .
$$

This "touchdown" flux is evaluated at the moment when the photosphere has receded back to the neutron star surface (see also Ref. [3]). In the above equation, $k_{\mathrm{es}}=$ $0.2(1+X) \mathrm{cm}^{2} \mathrm{~g}^{-1}$ is the electron scattering opacity in the stellar atmosphere. In the atmospheric models, we considered a wide range of hydrogen mass fraction $X$ consistent with the properties of each binary system.

Combining the measurements of $A$ and $F$ with the distance $D$ to each source, we obtain tight, uncorrelated constraints on the masses and radii of neutron stars. We have applied this technique to three sources, the neutron stars in the binaries 4U 1608-248, EXO 1745-248,

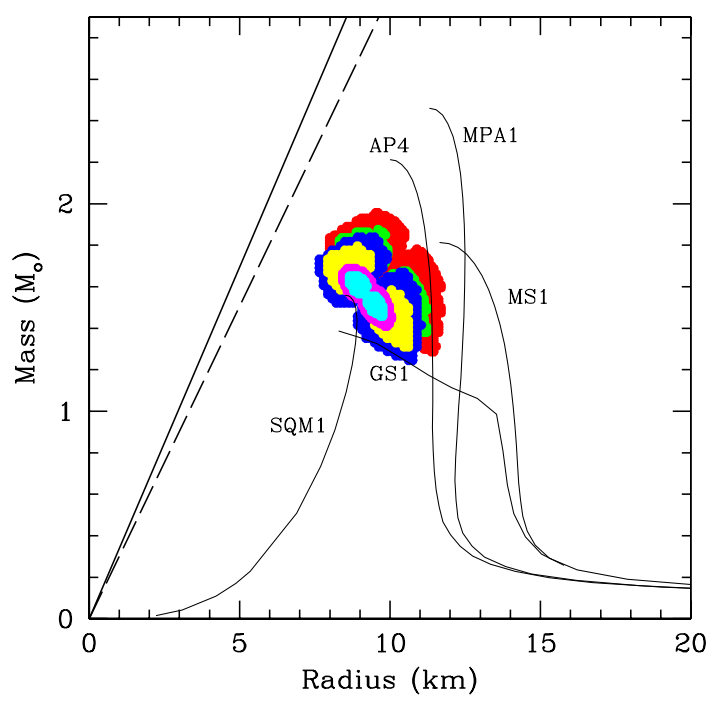

FIG. 1: The 1- and 2- $\sigma$ confidence contours for the masses and radii of three neutron stars in the binaries $4 \mathrm{U} 1608-248$ (green/red), EXO 1745-248 (yellow/blue), and 4U 1820-30 (cyan/magenta), compared with predictions of representative EoS (see text for details). The details of the measurements are described in Refs. 3]. The diagonal lines are the black-hole event horizon (solid) and Buchdahl (dashed) 25] limits.

and $4 \mathrm{U} 1820-30$, and show in Fig. 1 the $1-$ and $2-\sigma$ confidence contours of their masses and radii determinations [3]. The results are a set of uncorrelated measurements of neutron star masses and radii. (An earlier measurement of the mass and radius of the neutron star in EXO 0748-676 23] was based on the identification of atomic line features in its X-ray spectrum with gravitationally redshifted lines from its surface, which has since been shown to be inconsistent [27] with the recent measurement of its rapid spin frequency [28]).

The measurements in Fig. 1 incorporate the corrections and systematic uncertainties associated with the modeling of emission from the hot surfaces of neutron stars following a thermonuclear burst, the composition of the neutron star surface, as well as statistical or systematic uncertainties in the distances to the binaries. In all three measurements, uncertainties arising from subtraction of the background flux are negligible, because in each source, the luminosity from the neutron star surface exceeds the accretion luminosity by more than a factor of ten. Since not every combination of observables leads to a solution for $M$ and $R$, we converted the probability densities over the measured fluxes, apparent areas, and distances to those over the neutron star mass and radius following standard Bayesian statistics [3]. As a result, the uncertainties in the mass and radius, of order $15 \%$, are smaller than those of the individual spectroscopic quantities. Note that the confidence contours shown in Fig. 1 correct a small numerical error in the Jacobian transformation of Refs. [3].

As noted, three distinct measurements of neutron star 


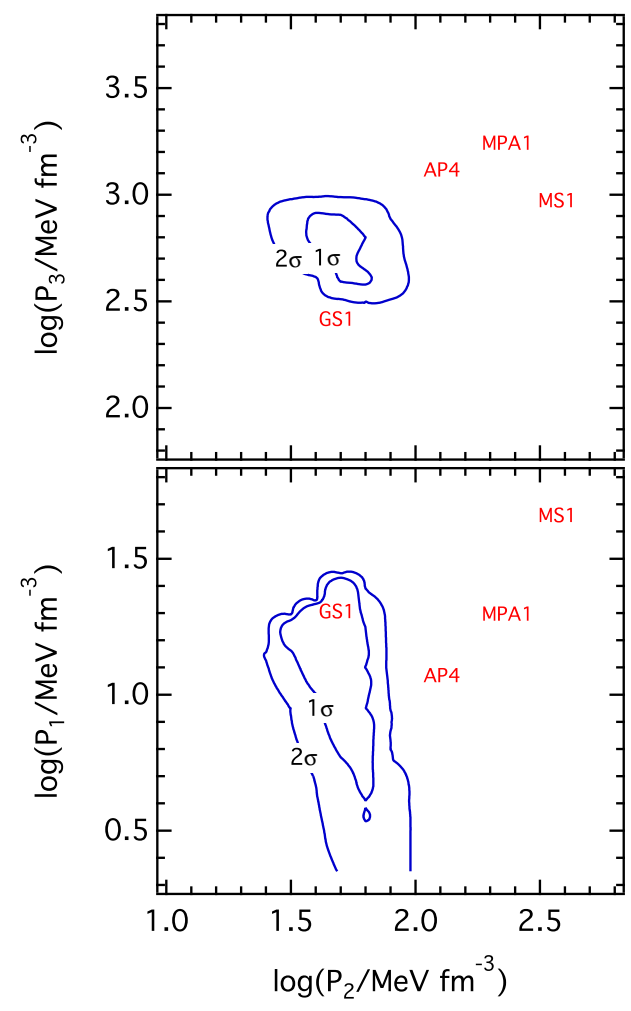

FIG. 2: The pressure of cold matter at (top) 7.4 and $3.7 \rho_{\mathrm{ns}}$ and (bottom) 1.85 and $3.7 \rho_{\text {ns }}$.

masses and radii allow us to infer a piecewise EoS of matter at supranuclear densities [19]. This approach makes the explicit assumption that the density in the neutron star surface layers smoothly reduces to zero, and is, therefore, not applicable to strange quark matter stars that are not gravitationally bound. In particular, we follow the procedure in Ref. [19] to convert these measurements to probability densities over the pressures of neutron star matter at three fiducial baryon densities $\rho_{1}=1.85 \rho_{\mathrm{ns}}$, $\rho_{2}=3.7 \rho_{\mathrm{ns}}$, and $\rho_{3}=7.4 \rho_{\mathrm{ns}}$. In these calculations, we include the full probability density for all three sources. We supplement this procedure with the requirement of causality, rejecting combinations of pressures for which the sound speed is larger than the speed of light [19]. Fig. 2 shows the confidence contours of different pairs of pressures (integrated over the third pressure).

The pressure at $\rho_{1}$ is only weakly constrained because such low densities are important only in determining the macroscopic properties of neutron stars with masses smaller than those in our measured sample [19]. In contrast, the pressures at $\rho_{2}$ and $\rho_{3}$ are constrained to within a factor of $\sim 10^{0.3}$.

In detail, we describe these data with a phenomenological piecewise polytropic EoS above density $\rho_{0}=$ $10^{14} \mathrm{~g} \mathrm{~cm}^{-3}$, which we smoothly connect to the SLy EoS at lower densities [26]. Between $\rho_{0}$ and $\rho_{4}$ we fit the energy density in the interval $\rho_{i-1}<\rho \leq \rho_{i}$ as
TABLE I:

Measured pressures at three supranuclear densities, in $\mathrm{MeV} \mathrm{fm}{ }^{-3}$, together with $P_{0}$ taken from the low density SLy EoS [26].

\begin{tabular}{|c|c|c|c|}
\hline $\log P_{0}\left(0.37 \rho_{\mathrm{ns}}\right)$ & $\log P_{1}\left(1.85 \rho_{\mathrm{ns}}\right)$ & $\log P_{2}\left(3.7 \rho_{\mathrm{ns}}\right)$ & $\log P_{3}\left(7.4 \rho_{\mathrm{ns}}\right.$ \\
\hline-0.64 & {$[0.6-1.4]$} & $1.70_{-0.15}^{+0.15}$ & $2.8_{-0.2}^{+0.1}$ \\
\hline
\end{tabular}

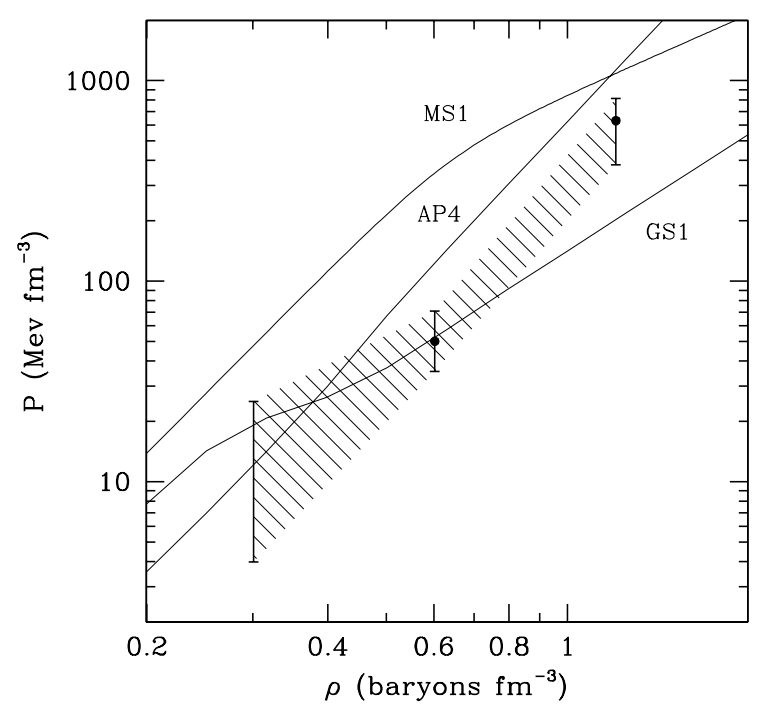

FIG. 3: Pressure vs. baryon density at the three points, $P_{1}$, $P_{2}$, and $P_{3}$, together with the fitted EoS (3). The shaded region shows the uncertainties in the determination.

$\epsilon=\alpha_{i} \rho+\beta_{i} \rho^{\Gamma_{i}}$, from which we derive the pressure, $P=\rho^{2} \partial(\epsilon / \rho) / \partial \rho=\left(\Gamma_{i}-1\right) \beta_{i} \rho^{\Gamma_{i}}$. Since the TolmanOppenheimer-Volkoff equation relates $\epsilon$ and $P$ to the mass and radius of the star, one only can determine the baryon density $\rho$ from $\epsilon$ and $P$ data to within a scale factor; to determine the scale we connect our fit to the tabulated low density SLy EoS. For $\rho_{i-1}<\rho \leq \rho_{i}$, the effective polytropic index is $\Gamma_{i} \equiv \log \left(P_{i} / P_{i-1}\right) / \log \left(\rho_{i} / \rho_{i-1}\right)$, the pressure is

$$
P=P_{i}\left(\frac{\rho}{\rho_{i}}\right)^{\Gamma_{i}},
$$

and in the energy density

$$
\alpha_{i}=\frac{\epsilon_{i-1}}{\rho_{i-1}}-\frac{P_{i}}{\left(\Gamma_{i}-1\right) \rho_{i-1}}\left(\frac{\rho_{i-1}}{\rho_{i}}\right)^{\Gamma_{i}} .
$$

Table 1 shows the fitting parameters. For $\rho>\rho_{3}$, we extrapolate the last polytropic relation.

The data present a clear challenge to microscopic nuclear calculations. Figs. 2 and 3 compare the best fit values of the pressures at the three fiducial densities with those predicted by a representative sample of EoS based on a wide range of input physics and computational methods, from nucleonic: variational chain summation with the AV18 potential, UIX three-body potential plus 
relativistic boost corrections $A P_{4}[8]$; Dirac-BruecknerHartree-Fock MPA1 15]; and relativistic mean fields $M S 1$ 14], plus kaons GS1 13]. (Although our procedure is not applicable to the u,d,s quark matter SQM1 [15], its predictions can nevertheless be compared directly to the individual mass and radius measurements shown in Fig. 1). Our measurements clearly discriminate between different predictions, and indicate that the EoS based on nucleons alone, AP4, MP1 and MS1, are too stiff at higher density - a conclusion also borne out by the comparisons of $M$ vs. $R$ in Fig. 1 which show that the predicted radii at the measured masses are too large. As one sees clearly in Fig. 3, the data call for a softer EoS, as would be produced by including degrees of freedom beyond nucleons, e.g., hyperons, mesons, and quarks, or possibly produced by a better description of nucleonic interactions. In particular a softer EoS, by allowing larger central densities than an EoS with only two and three body nucleonic interactions (e.g., AP4 with a central density $\sim 7 \rho_{n s}$ at the maximum mass $\sim 2.2 M_{\odot}$ ) would, in the naive picture of a sharp phase transition from nucleonic to quark matter, allow quark cores to appear in more massive stars.

The allowed range of pressures seen in Fig. 3 also reduces the maximum neutron star mass compared to that predicted by purely nucleonic EoS. It is, nevertheless, consistent with the recent measurement of a $1.97 \pm 0.04 M_{\odot}$ neutron star by observations of Shapiro delay [29].

Our measurements can be confirmed or tested by additional mass-radius measurements in bursting sources, improved distance measurements by space-based interferometers, and more definitively by observations of other phenomena that probe the masses and radii of neutron stars such as gravitationally redshifted absorption lines or flux oscillations that depend on surface gravity.

\section{Acknowledgements}

F. Ö. thanks the Institute for Theory and Computation at Harvard University for their hospitality. We gratefully acknowledge useful conversations with A. Loeb, R. Narayan, D. Psaltis and K. Rajagopal. F. Ö. acknowledges support from NSF grant AST-07-08640 and Chandra Theory grant TMO-11003X. This research was supported in part by NSF Grant PHY-07-01611.
[1] M. A. Stephanov (2006) arXiv:hep-lat/0701002; S. Hands Contemp. Phys. 42, 209 (2001) arXiv:physics/0105022

[2] G. Baym, T. Hatsuda, M. Tachibana, \& N. Yamamoto, J. Phys. G: Nucl. Part. Phys. 35 (2008) 104021; arXiv:0806.2706.

[3] F. Özel, T. Güver, D. Psaltis Astrophys. J., 693, 1775 (2009); T. Güver, F. Özel, A. Cabrera-Lavers, \& P. Wroblewski, Astrophys. J., 712, 964 (2010); T. Güver, P. Wroblewski, L. Camarota, \& F. Özel, Astrophys. J., in press (2010) arXiv:1002.3825

[4] C. Fryer, Astrophys. J., 522, 413 (1999); F. D. Swesty, J. M. Lattimer, E. S. Myra, Astrophys. J., 425, 195 (1994).

[5] V. Kalogera and G. Baym, Ap. J. Letters 470, L61 (1996)

[6] A. I. MacFadyen, S. E. Woosley, \& A. Heger Astrophys. J. 550, 410 (2001)

[7] L. Baiotti, B. Giacomazzo, \& L. Rezzolla, Phys. Rev. D, 78, 4033 (2008); M. Bejger et al. Astron. \& Astrophys. 431, 297 (2005)

[8] A. Akmal, V.R. Pandharipande, \& D.G. Ravenhall, Phys. Rev. C 58, 1804 (1998).

[9] J. Morales, Jr., V.R. Pandharipande, \& D.G. Ravenhall, Phys. Rev. C 66, 054308 (2002).

[10] A. Mukherjee, Phys. Rev. C 79,045811 (2009).

[11] V. R. Pandharipande, C. J. Pethick, \& V. Thorsson, Phys. Rev. Lett. 75, 4567 (1995).

[12] H.A. Bethe \& G.E. Brown, G.E. Brown, Astrophys. J. 506, 780 (1998), and references therein.

[13] N.K. Glendenning \& J. Schaffner-Bielich, Phys. Rev. C60, 025803 (1999).

[14] H. Müller \& B.D. Serot, Nucl. Phys., A606 (1996).

[15] J. M. Lattimer \& M. Prakash, Astrophys. J., 550, 426 (2001); J. M. Lattimer \& M. Prakash, Phys. Rep., 442, 109 (2007)

[16] S. E. Thorsett \& D. Chakrabarty ApJ, 512, 288 (1999)
[17] B. Link, R. I. Epstein, \& J. M. Lattimer, Phys. Rev. Lett., 83, 3362 (1999)

[18] Heinke, C. O., Rybicki, G. B., Narayan, R., \& Grindlay, J. E. Astrophys. J., 644, 1090 (2006); Webb, N. A. \& Barret, D., Astrophys. J. 671, 727 (2007); Rutledge, R. E., Bildsten, L., Brown, E. F., Pavlov, G. G., Zavlin, V. E., Ushomirsky, G., Astrophys. J. 580, 413 (2002); S. Bogdanov, G. B. Rybicki, \& J. E. Grindlay, Astrophys. J., 670, 668 (2007); W. Ho \& C. O. Heinke, Nature, 462, $71(2009)$

[19] F. Özel \& D. Psaltis, Phys. Rev. D, 80, 103003 (2009)

[20] J. S. Read, B. D. Lackey, B. J. Owen, \& J. L. Friedman, Phys. Rev. D 79, 124032 (2009)

[21] D. Galloway, M. Muno, J. Hartman, P. Savov, D. Psaltis, \& D. Chakrabarty, Astrophys. J. Suppl., 179, 360 (2008)

[22] A. M. Juett, N. S. Schulz, \& D. Chakrabarty, Astrophys. J., 612, 308 (2004); A. M. Juett, N. S. Schulz, D. Chakrabarty, \& T. W. Gorczyca, Astrophys. J., 648, 1066 (2006)

[23] F. Özel, Nature, 441, 1115 (2006)

[24] J. van Paradijs, Nature, 274, 650 (1978); J. van Paradijs, Astrophys. J., 234, 609 (1979)

[25] H. A. Buchdahl, Phys. Rev., 116, 1027 (1959)

[26] F. Douchin, \& P. Haensel, Astron. \& Astrophys., 380, $151(2001)$

[27] J. Lin, F. Özel, D. Chakrabarty, \& D. Psaltis, Astrophys. J., in review (2010) arXiv:1007.1451

[28] D. K. Galloway, J. Lin, D. Chakrabarty, D., \& J. M. Hartman, Astrophys. J., 711, L148 (2010)

[29] P. Demorest, T. Pennucci, S. Ransom, M. Roberts, \& J. Hessels, Nature, 467, 1081 (2010); F. Özel, D. Psaltis, S. Ransom, P. Demorest, \& M. Alford, Ap. J. Lett. 724, 199 (2010) 